\title{
Transformation of human lymphocytes in vitro by autologous and allogeneic rheumatoid synovial fluids
}

\author{
T. DOUGLAS KINSELLA \\ From McGill University Rheumatic Diseases Unit, and Department of Medicine, \\ Royal Victoria Hospital, Montreal, Quebec, Canada
}

\begin{abstract}
Kinsella, T. D. (1976). Annals of the Rheumatic Diseases, 35, 8-13. Transformation of human lymphocytes in vitro by autologous and allogeneic rheumatoid synovial fluids. To assess the possible participation of cellular immune mechanisms in the pathogenesis of rheumatoid arthritis (RA) in vitro studies of the blastogenicity of rheumatoid and nonrheumatoid synovial fluids for human peripheral blood lymphocytes were conducted. In autologous cultures it was found that 13 of 19 rheumatoid fluids induced significant lymphocyte blastogenesis, whereas only 1 of 13 nonrheumatoid fluids induced such a response. In allogeneic cultures rheumatoid fluids induced significant blastogenesis of RA lymphocytes in 18 of 23 experiments, and of non-RA lymphocytes in 8 of 18 experiments. By contrast, nonrheumatoid fluids induced significant blastogenesis of RA lymphocytes in 2 of 13 experiments, and of non-RA lymphocytes in 1 of 14 experiments. The blastogenicity of fluids was found to correlate significantly with the presence therein of immunofluorescent intracellular inclusions of immunoglobulin and complement. These studies support the concept that the presence of immune complexes in the majority of rheumatoid synovial fluids might render the latter blastogenic for human lymphocytes in vivo, thereby perpetuating rheumatoid synovitis.
\end{abstract}

Evidence from various sources (Ziff, 1973) indicates that immune mechanisms are involved in the pathogenesis of intra-articular inflammation in patients with rheumatoid arthritis (RA). Since the effector limbs of immune inflammation can be partitioned into humoral and/or cell-mediated mechanisms (Craddock, Longmire, and McMillan, 1971), it has been convenient for investigators to evaluate the relative contributions of each of these events to rheumatoid synovial inflammation. As a result, certain observations have been made which clearly document the participation of humoral immune inflammation in RA, including the demonstration of intrasynovial synthesis of immunoglobulin (Ig) and rheumatoid factor (Smiley, Sachs, and Ziff, 1968; Sliwinski and Zvaifler, 1970), the presence of immune complex-like inclusions in intra-articular phagocytic cells (Hollander and others, 1965; Kinsella, Baum, and Ziff, 1969), and the intra-articular activation and consumption of components of the complement (C) system (Ruddy, Gigli, and Austen, 1972).
Only recently has the participation of cell-mediated events in the pathogenesis of rheumatoid synovitis been definitively documented, showing that such participation may occur when RA synovial fluids (Stastny and others, 1973) and RA synovial extracts (Stanfield and others, 1967; Bacon and others, 1973) are used as in vitro stimulants. Previous work by the author has shown that the majority of RA synovial fluids can induce in vitro blastogenic transformation of autologous peripheral blood lymphocytes from subjects with RA (Kinsella, 1973). The present study extends this observation and also evaluates the induction of in vitro blastogenesis by allogeneic synovial fluids as well as examines various in vivo and in vitro correlates of the reaction.

\section{Materials and methods STUDY GROUPS}

Donors of peripheral blood leucocytes, synovial fluid, or both, are given in Table I. All patients with RA had 
Table I Diagnosis of donors of cells and synovial fluids $(S F)$ used in cultures

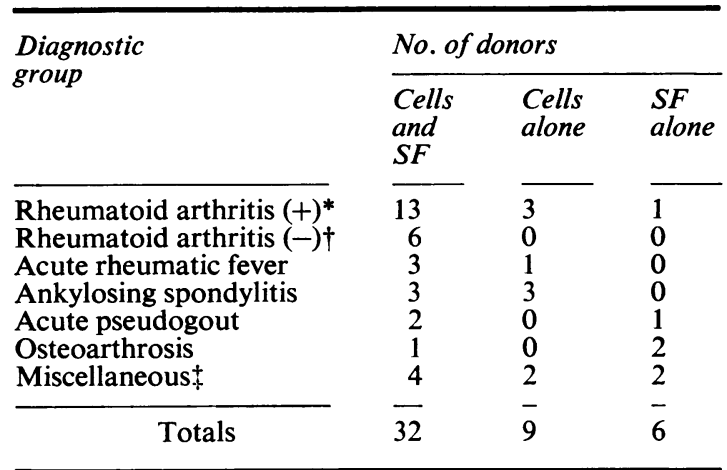

$*(+)=$ positive for rheumatoid factor; $+(-)=$ negative for rheumatoid factor: + miscellaneous: cells and SF = gout, traumatic arthritis, villonodular synovitis, and transient polyarthritis of unknown aetiology; cells alone = variable immune deficiency and normal; SF alone = psoriatic arthritis and subacute bacterial endocarditis.

'classical' or 'definite' disease, as defined by American Rheumatism Association criteria. Patients with ankylosing spondylitis met diagnostic criteria for 'definite' disease (Bennett and Burch, 1967). All other patients had definitive diagnoses derived from the clinical as well as from appropriate biochemical, serological, radiological, and histological examinations. Thirty-two patients donated both lymphocytes and synovial fluid, nine donated lymphocytes alone, and six synovial fluid alone.

At the time of these studies all RA patients were receiving acetylsalicylic acid therapy $1200-4800 \mathrm{mg} /$ day; six were receiving 5-10 mg prednisone per day; two were receiving $250 \mathrm{mg}$ antimalarials per day; and two received therapeutic doses of gold salts. In the groups without RA (non-RA) the majority were receiving 1200-3600 $\mathrm{mg}$ acetylsalicylic per day during acute exacerbations of synovitis, and eight were receiving a maximum of $400 \mathrm{mg}$ phenylbutazone per day; six patients in this group were receiving no anti-inflammatory medication.

\section{SEROLOGICAL STUDIES}

Sera and/or synovial fluids of all patients were examined for the presence of rheumatoid factor (RF) by a slide latex test (Gibco-Grand Island Biologicals) and a micromethod modification of a sensitized sheep cell test, employing reagents donated by the World Health Organization (Anderson and others, 1970). Thirteen patients with RA were positive for RF and six were negative. Of the non-RA group only one synovial fluid donor with osteoarthrosis was positive for RF.

Direct immunofluorescent staining was performed on washed cells recovered from aliquots of 30 of 38 synovial fluids (RA $\cdot R F(+) 11, R A \cdot R F(-) 5$, rheumatic fever 3, ankylosing spondylitis 3 , pseudogout 3 , osteoarthrosis 2 , miscellaneous 3), as described previously (Kinsella and others, 1969). Cytocentrifuge-prepared smears were stained to detect the presence of immunofluorescent inclusions of $\mathrm{IgG}, \mathrm{A}$, and $\mathrm{M}$ and the third component of complement (C3). Positive preparations were arbitrarily considered to be those in which at least $25 \%$ of cells in five fields contained more than five inclusions per cell.
Fluorescein-conjugated antisera to polyvalent human Ig and C3 were obtained from Behring Diagnostics.

\section{PREPARATION OF SYNOVIAL FLUID (SF)}

Aspiration of SF was performed under sterile conditions. Aliquots of specimens were used for cytological, serological, and biochemical studies and the remainder collected in sterile polypropylene centrifuge tubes. In early studies centrifugation of SF was carried out at $26860 \times g$ at $4^{\circ} \mathrm{C}$ for 60 minutes in a JS-13 swinging bucket rotor in a Beckman J-21 centrifuge; subsequently it was found that centrifugation at $5720 \times g$ was adequate and did not influence the blastogenicity of SF. After centrifugation, the upper one-half of each specimen was removed under sterile conditions and aliquots were diluted in volumes of heat inactivated $\left(56^{\circ} \mathrm{C} \times 30 \mathrm{~min}\right)$ gamma globulin-free newborn calf serum (GG-Free Newborn Calf Serum, Gibco) sufficient to provide final dilutions of SF of $1 / 10$, $1 / 20$, and $1 / 40$. Specimens were stored at $-20^{\circ} \mathrm{C}$ until used in leucocyte cultures.

\section{LEUCOCYTE CULTURES}

$50 \mathrm{ml}$ of venous blood, containing $10 \mu \mathrm{m} / \mathrm{ml}$ heparin (Connaught Laboratories), were obtained from each cell donor. Red blood cells were usually sedimented by gravity at room temperature in an inverted syringe but in some instances several centrifugations at $75 \times g$ were necessary to obtain adequate sedimentation of the red blood cells. The supernatant plasma thus obtained was centrifuged in glass tubes at $250 \times g$ for 10 minutes and the resultant cell button washed three times in RPMI-1640 medium (Gibco) before resuspension in an amount of RPMI sufficient to obtain $5 \times 10^{5}$ small lymphocytes in $0.2 \mathrm{ml}$.

Leucocytes were cultured in polypropylene tubes (Falcon Plastics) in triplicate or quadruplicate in final volumes of $1.0 \mathrm{ml}$, containing $0.2 \mathrm{ml}$ cell suspension, RPMI-1640 medium, 1\% $200 \mathrm{mmol} / \mathrm{l}$ L-glutamine (Gibco), antibiotics, and $20 \%$ of either heat inactivated calf serum alone (control cultures) or SF diluted in calf serum (experimental cultures); final dilutions of SF in each culture were $1 / 50,1 / 100$, and $1 / 200$. In the majority of experiments parallel cultures of both RA SF and non-RA SF were included with lymphocytes from a single donor. Additional cultures containing phytohaemagglutinin (PHA-P, Difco) were established for all RA patients and for all but two non-RA patients; in these experiments PHA was added on day 0 for 3-day cultures and on day 3

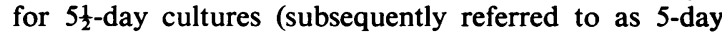
cultures).

Heat inactivated gamma globulin-free newborn calf serum (AGF), which was devoid of bovine C3 by Ouchterlony analysis (Ouchterlony, 1962), was used as carrier and diluent serum since preliminary comparisons with a variety of serum supplements showed that AGF was less likely to induce 'spontaneous' blastogenesis of unstimulated cultures. Cultures were routinely maintained for 5 days, although in some experiments parallel cultures were maintained for both 3 days and 5 days to ascertain the effect of duration of culture. Individual SF were tested against autologous lymphocytes obtained at several different times when possible.

Eighteen hours before harvesting cultures were pulsed with $2 \mu \mathrm{Ci}$ tritiated thymidine $\left({ }^{3} \mathrm{HTdr}\right.$-Amersham Searle, sp. act. 5.0 Ci/mmol/1 (and processed for liquid scintilla- 


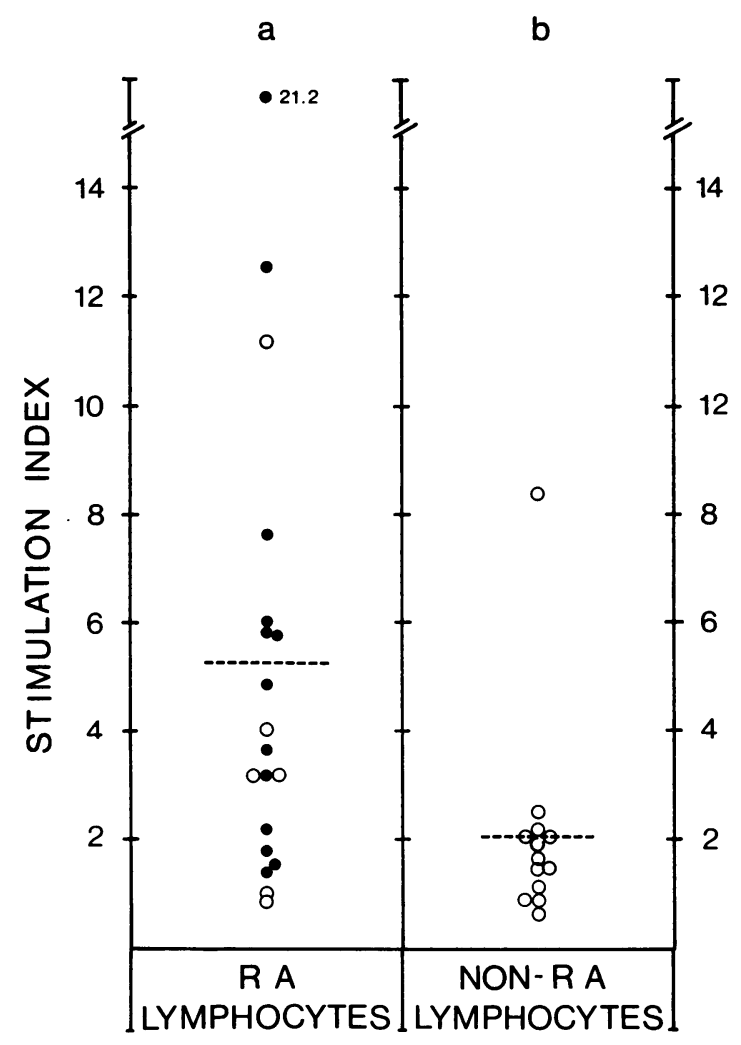

FIG. 1 Stimulation indices (mean cpm of cultures containing $S F$ divided by mean cpm of cultures without $S F)$ of 19 $R A$ and 13 non-RA subjects in presence of autologous lymphocytes and synovial fluids. - represents $S F$ positive for rheumatoid factor, OSF negative for rheumatoid factor

tion counting in a Phillips liquid scintillation counter as reported previously (Kinsella, 1973). Results are expressed as a stimulation index, which is the ratio obtained from mean counts per minute in replicate experimental cultures (containing SF) divided by mean cpm in replicate control cultures (containing no SF). A stimulation index of 3.0 or greater was arbitrarily considered as significant. In each experiment the maximum stimulation index induced by one of the three dilutions of SF used was considered to represent the blastogenicity of the SF. Statistical comparison of mean stimulation index was carried out by Student's ' $t$ ' test using an Olivetti Programma 101 calculator; $\chi^{2}$ analysis was used to compare blastogenicity, the presence of rheumatoid factor, and the presence of Ig- and C-containing cells in SF.

\section{Results}

BLASTOGENICITY OF SF FOR AUTOLOGOUS LYMPHOCYTES

Fig. 1 shows that the mean stimulation index (SI) of 19 RA subjects with autologous SF was $5 \cdot 3 \pm 1 \cdot 1$, range $0 \cdot 9-21 \cdot 2 ; 13$ of the 19 subjects ( $9 R A+, 4 R A-)$ gave an SI $>3 \cdot 0$. The mean SI of 13 non-RA subjects with autologous SF was $2 \cdot 1 \pm 0 \cdot 5$, range $0 \cdot 6-8 \cdot 3$; only $\frac{\text { गु }}{\sigma}$ one subject, with pseudogout, had an SI $>3 \cdot 0$. The difference between the means of the two groups was significant $(P<0.02)$.

BLASTOGENICITY OF RA SF FOR ALLOGENEIC LYMPHOCYTES

The SI of 14 RA SF used in 23 experiments with 18 으 allogeneic RA lymphocyte donors are shown in $\frac{\bar{\sigma}}{\sigma}$ Fig. $2 a$. The mean SI was $5 \cdot 3 \pm 0 \cdot 8$, range $0 \cdot 7-14 \cdot 3 ; \stackrel{\infty}{\circ}$ 18 of the 23 RA SF gave an SI $>3.0$. In Fig. $2 b$ are shown the SI produced by 12 RA SF used in 18 experiments with 14 allogeneic non-RA lymphocyte? donors. The mean SI in these experiments was $\vec{\omega}$ $4 \cdot 3 \pm 0 \cdot 8$, range $0 \cdot 2-13 \cdot 3 ; 7$ of the 12 RA SF gave an $\stackrel{\sigma}{\circ}$ SI $>3.0$. The difference between the means of the SI induced by RA SF with allogeneic RA and non-RA lymphocyte donors was not significant $(P>0.05)$.

BLASTOGENICITY OF NON-RA SF FOR

ALLOGENEIC LYMPHOCYTES

The SI of 10 non-RA SF used in 13 experiments with $\overrightarrow{-}$ 12 allogeneic RA lymphocyte donors can be seen in $\mathbb{D}$

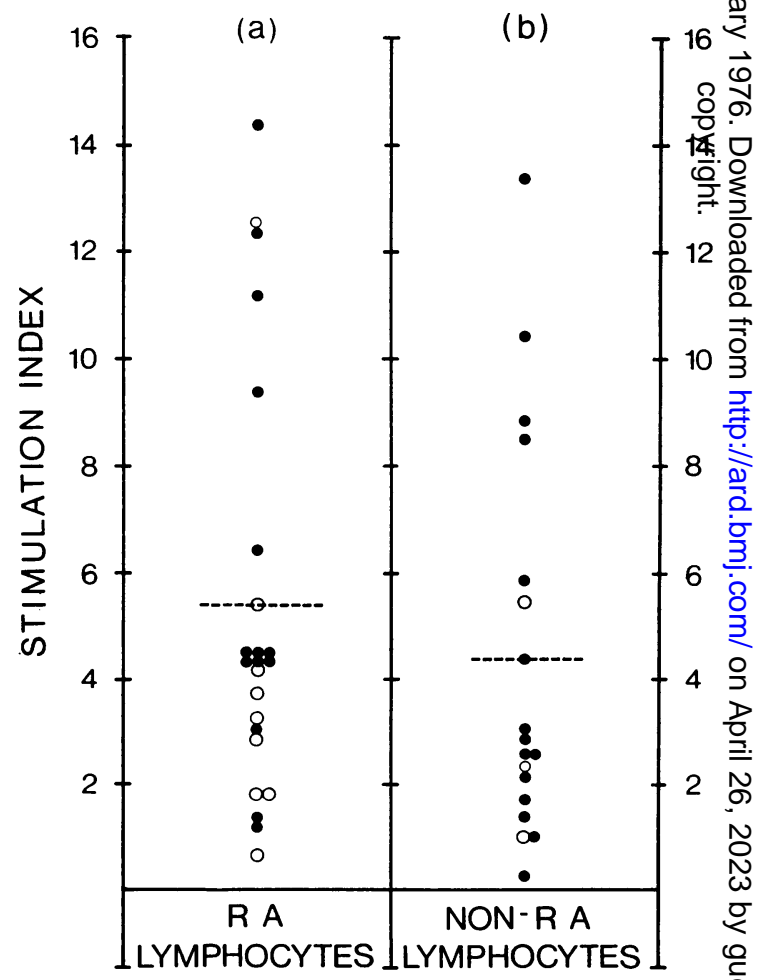

FIG. 2 Stimulation indices of $R A S F$ cultured with allo- $\$$ geneic lymphocytes from $18 R A$ cell donors and 14 non- $R A$ cell donors. In (a) $14 R A S F$ were used in 23 experiments. In (b) 12 RA SF were employed in 18 experiments. $\bullet$ represents $S F$ positive for rheumatoid factor, OSF negative for $\bigcirc$ rheumatoid factor 


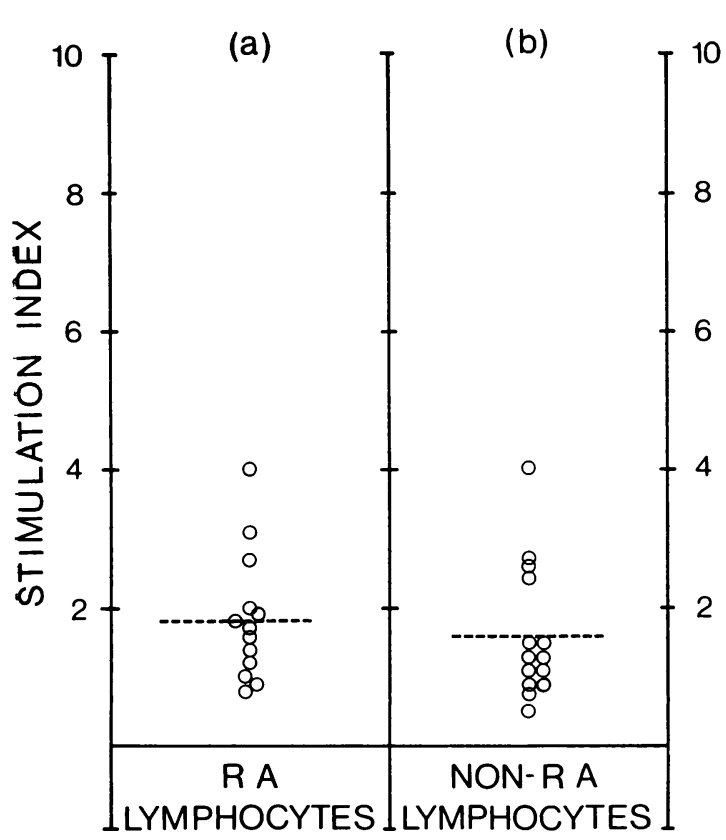

FIG. 3 Stimulation indices of non-RA SF cultured with allogeneic lymphocytes from $12 R A$ cell donors and 9 non-RA donors. In (a) 10 non- $R A$ SF were used in 13 experiments. In (b) 11 non-RA SF were used in 14 experiments

Fig. $3 a$. In these experiments the mean SI was $1 \cdot 8 \pm$ $0 \cdot 3$, range $0 \cdot 8-4.0$; SF from one patient each with pseudogout and OA gave an SI of 4 and 3, respectively.

In Fig. $3 b$ are shown the SI produced by 11 nonRA SF in 14 experiments with 9 allogeneic non-RA lymphocyte donors. The mean SI in these experiments was $1 \cdot 6 \pm 0 \cdot 6$, range $0 \cdot 5-4 \cdot 0$; SF from one patient with pseudogout, who was not the same patient noted in Fig. $3 a$, gave an SI of 4 . The difference between the means of the SI induced by non-RA SF with allogeneic RA and non-RA lymphocyte donors was not significant $(P>0.05)$.

PHA RESPONSES IN 5-DAY CULTURES

The mean SI for 19 RA lymphocyte donors in response to PHA added after 2 days of culture was $22 \cdot 1 \pm 5 \cdot 4$, range $1 \cdot 1-101 \cdot 1$. For 12 non-RA lymphocyte donors the mean SI was $18 \cdot 6 \pm 4 \cdot 7$, range $1 \cdot 0$ 47.8; the difference between these means was not significant $(P>0.05)$, indicating that RA lymphocytes were not nonspecifically more sensitive than non-RA lymphocytes to in vitro blastogenic stimuli.

EFFECT OF DURATION OF CULTURE ON SF-INDUCED BLASTOGENESIS

The blastogenic effect of 6 RA SF and 6 non-RA SF in parallel cultures harvested after 3 days and 5 days were evaluated (data not shown). It was found that in both autologous and allogeneic 3-day cultures no significant lymphocyte blastogenesis occurred; in no instance was an SI $>2$ observed. On the other hand, significant blastogenesis $(\mathrm{SI} \geqslant 3)$ was frequently observed in 5-day cultures, regardless of the magnitude of the SI at day 3 in both autologous and allogeneic RA cultures.

\section{COMPARATIVE BLASTOGENICITY OF SF FOR}

\section{ALLOGENEIC LYMPHOCYTES}

Representative examples which compare the blastogenicity of both RA SF and non-RA SF for multiple allogeneic lymphocyte donors are listed in Table II. Although there was some variation in the range of SI induced by single fluids, it can be seen that there was nevertheless good reproducibility of the blastogenicity of individual SF for allogeneic lymphocytes. Thus, 'positive' $S F(S I \geqslant 3)$ were usually positive for multiple cell donors, whereas 'negative' SF were negative for multiple cell donors.

\section{INFLUENCE OF DILUTION OF SF ON} BLASTOGENIC RESPONSES

Table III summarizes the results of the effect of dilution of SF in RA and non-RA autologous and allogeneic cultures. It can be seen that, of 19 autologous RA SF, 6 induced significant blastogenesis at one dilution of SF, 4 at two dilutions, and 3 at three dilutions, while 6 were 'negative' at all three dilutions. By contrast, of 13 autologous non-RA SF, one pseudogout fluid induced significant blastogenesis at one dilution while all other fluids failed to induce significant blastogenesis at any dilution.

In allogeneic cultures of 23 RA SF with RA lymphocytes, significant blastogenesis was induced by 8 fluids at one dilution, by 5 at two dilutions, and by 5 at three dilutions, whereas 5 failed to induce

Table II Comparison of blastogenicity of individual synovial fluids for allogeneic lymphocyte donors

\begin{tabular}{|c|c|c|}
\hline \multirow{2}{*}{$\begin{array}{l}\text { Diagnosis* of } \\
\text { synovial fluid donor }\end{array}$} & \multirow{2}{*}{$\begin{array}{l}\text { SI† of } \\
\text { lymphocyte donors }\end{array}$} & Times positive $\ddagger$ \\
\hline & & Times employed \\
\hline $\begin{array}{l}\text { RA(+) } \\
\text { RA(+) } \\
\text { RA(+) } \\
\text { RA(-) } \\
\text { RA(-) } \\
\text { Rheumatic fever } \\
\text { Ankylosing spondyli } \\
\text { Osteoarthrosis } \\
\text { Traumatic arthritis }\end{array}$ & $\begin{aligned} 4 \cdot 3,6 \cdot 4,9 \cdot 4,34 \cdot 2 \\
3 \cdot 0,4 \cdot 5 \\
2 \cdot 5,5 \cdot 8,13 \cdot 3 \\
1 \cdot 6,4 \cdot 2 \\
0 \cdot 7,1 \cdot 8 \\
\quad 0 \cdot 8,2 \cdot 4 \\
\quad 1 \cdot 1,1 \cdot 3 \\
1 \cdot 5,2 \cdot 6 \\
1 \cdot 4,1 \cdot 7,1 \cdot 9\end{aligned}$ & $\begin{array}{l}4 / 4 \\
2 / 2 \\
2 / 3 \\
1 / 2 \\
0 / 2 \\
0 / 2 \\
0 / 2 \\
0 / 2 \\
0 / 3\end{array}$ \\
\hline
\end{tabular}

* RA (+) and RA $(-)=$ rheumatoid arthritis, positive and negative for rheumatoid factor.

$+\mathbf{S I}=$ stimulation index (mean cpm of cultures containing synovial fluid divided by mean cpm of cultures containing no synovial fluid); each value derived from a different lymphocyte donor.

$\mp$ Positive $\geqslant 3 \cdot 0$. 
significant blastogenesis at any dilution. When 18 allogeneic RA SF were cultured with non-RA lymphocytes 2 induced significant blastogenesis at one dilution and 6 at three dilutions, whereas 10 failed to induce blastogenesis at any dilution tested.

In cultures of allogeneic non-RA SF, 13 fluids were tested against RA lymphocytes and 2 were 'positive' at one dilution, while all others failed to induce significant blastogenesis at any dilution. Similarly, when 14 allogeneic non-RA SF were cultured with non-RA lymphocytes one fluid induced significant blastogenesis at two dilutions, whereas 13 were 'negative' at all dilutions employed.

In three experiments with autologous cultures (2 RA, 1 non-RA) and in five with allogeneic cultures (3 RA SF, 2 non-RA SF) using extended dilutions ranging from undiluted to $1 / 5000$, no appreciable change in the frequency of significant blastogenesis was encountered (data not shown).

\section{INFLUENCE OF THERAPY ON BLASTOGENIC RESPONSE}

No inhibitory effect of drug therapy was evident from these studies. In particular, for patients with RA no correlation was found between type of therapy and quantitative blastogenic responses, including those on prednisone therapy of whom three displayed 'positive' and three 'negative' stimulation index.

\section{INFLUENCE OF IN VIVO CORRELATES ON SF BLASTOGENESIS}

Total and differential white blood cell counts did not correlate with either the frequency or degree of SF-induced blastogenesis. Similarly, the presence of RF in 13 of 32 SF did not correlate with the induction of blastogenesis $\left(\chi^{2}=2.9965\right.$; df $\left.1 ; P>0 \cdot 1\right)$. However, significant correlations were found between the presence of intracellular Ig and $\mathrm{C}$ inclusions and SF-induced blastogenesis. Of $30 \mathrm{SF}$ examined, intracellular Ig inclusions were present in 11 and absent in three with significant blastogenesis (SI $\geqslant 3$ ), whereas such inclusions were present in 5 and absent in 11 without significant blastogenesis $\left(\chi^{2}=4 \cdot 8469\right.$; df $1 ; P>0.05$ ). Similarly, immunofluorescent C3 inclusions were present in 11 and absent in $3 \mathrm{SF}$ with significant blastogenesis, but were present in 4 and absent in 12 without significant blastogenesis $\left(\chi^{2}=\right.$ 6.5624; df 1; P > 0.025).

\section{Discussion}

These studies extend a previous report from this laboratory that, in contrast to non-RA SF, the majority of RA SF can induce in vitro transformation of autologous peripheral blood lymphocytes (Kinsella, 1973). In addition, the results show that RA SF exert a quantitatively similar effect on allogeneic RA and non-RA lymphocytes. Thus, these studies indicate that RA SF can modulate a cell-mediated immune response in vitro, a conclusion supported by recent reports by other authors who used different techniques for assessing cellular immunity in vitro (Stastny and others, 1973; Bacon and others, 1973). Since lymphocyte transformation in vitro is a reliable correlate of cell-mediated immunity in vivo (Bloom Landy, and Lawrence, 1973), it can be inferred tha甲 the in vitro studies reported here may reflect similas events in vivo and partially explain the persisteris lymphoid proliferation observed in rheumatoi $\vec{A}^{+}$ synovial membranes.

The identity of the blastogenic substance, or substances, in RA SF has not been specifically delineated. However, the demonstration of a positive correlation between SF-induced blastogenesis and the presence in SF of immunofluorescent intracellular Ig and $\mathrm{C}$ inclusions, which are considered to represent phagocytosed immune complexes (Bonomo and others 1970; Ziff, 1973), suggests that the latter may

Table III Influence of dilution on blastogenicity of synovial fluids

\begin{tabular}{|c|c|c|c|c|c|c|}
\hline \multicolumn{2}{|c|}{ Diagnosis* of } & \multicolumn{5}{|c|}{ Synovial fluids } \\
\hline \multirow[t]{2}{*}{ Cell donor } & \multirow[t]{2}{*}{$S F$ donor } & \multirow[t]{2}{*}{$\begin{array}{l}\text { No. } \\
\text { used }\end{array}$} & \multicolumn{4}{|c|}{$\begin{array}{l}\text { No. of dilutions inducing } \\
\text { blastogenesis } \dagger\end{array}$} \\
\hline & & & 1 & 2 & 3 & None \\
\hline \multicolumn{7}{|c|}{ Autologous cultures } \\
\hline & - & 19 & 6 & 4 & 3 & 6 \\
\hline Non-RA & - & 13 & 1 & 0 & 0 & 12 \\
\hline \multicolumn{7}{|c|}{ Allogeneic cultures } \\
\hline RA & RA & 23 & 8 & 5 & 5 & 5 \\
\hline Non-RA & RA & 18 & 2 & 0 & 6 & 10 \\
\hline RA & Non-RA & 13 & 2 & 0 & 0 & 11 \\
\hline Non-RA & Non-RA & 14 & 0 & 1 & 0 & 13 \\
\hline
\end{tabular}


contribute to the observed response. Such an interpretation would also be consistent with a previous finding that immune complexes can induce 'nonspecific' lymphocyte transformation in vitro (BlochShtacher, Hirschhorn, and Uhr, 1968).

In addition, there are at least two other potential mitogens present in RA SF, namely, specific 'RA antigen' and aggregated gamma globulin. Although the former may contribute to the observed reaction, the absence of immunological specificity for RA lymphocytes indicates that SF-induced blastogenicity is not mediated exclusively by immune memory for such an antigen. On the other hand, the presence in some RA SF of aggregated gamma globulin (Hannestad, 1967), which can induce in vitro lymphocyte transformation (Reynolds and Abdou, 1973; Kinsella, 1974) and inhibition of leucocyte migration (Brostoff, Howell, and Roitt, 1973), suggests that the blastogenicity of RA SF may partially reflect the presence therein of aggregated gamma globulin.

The foregoing considerations and the results of the present studies suggest that the intra-articular milieu in patients with RA may be such that intrasynovial lymphocyte blastogenesis could occur under the influence of 'nonspecific', as well as specific, mitogens present in RA SF. Thus, in addition to their potential pathogenetic significance, these studies suggest that eradication or inhibition of intrasynovial lymphocyte blastogenesis is a rational therapeutic objective in the patient with rheumatoid arthritis.

The author gratefully acknowledges the technical assistance of Liliane LeBrun and Susan Sheldon, and the secretarial assistance of Kathy Peterson. These studies were supported by a grant from the Canadian Arthritis Society (CARS-6-142-70).

\section{References}

Anderson, S. G., Bentzon, M. W., Houba, V., And KraG, P. (1970) Bull. Wld Hlth Org., 42, 311 (International reference preparation of rheumatoid arthritis serum)

Bacon, P. A., Bluestone, R., Cracchiolo, A., AND Goldberg, L. S. (1973) Lancet, 2, 611 (Cell-mediated immunity to synovial antigens in rheumatoid arthritis)

BennetT, P. H., AND Burch, T. A. (1967) Bull. rheum. Dis., 17, 453 (New York symposium on population studies in rheumatic diseases: new diagnostic criteria)

Bloch-ShTACHeR, N., HiRSCHHORN, K., AND UHR, J. W. (1968) Clin. exp. Immunol., 3, 889 (The response of lymphocytes from non-immunized humans to antigen-antibody complexes)

Bloom, B. R., LANDY, M., and LaWrence, H. S. (1973) Cell. Immunol., 6, 331 (In vitro methods in cell-mediated immunity: a progress report)

Bonomo, L., TURSI, A., Trizio, D., Gillardi, U., AND Dammacco, F. (1970) Immunology, 18, 557 (Immune complexes in rheumatoid synovitis: a mixed staining immunofluorescence study)

Brostoff, J., Howell, A., AND RoItT, I. M. (1973) Clin. exp. Immunol., 15, 1 (Leukocyte migration inhibition with aggregated gamma globulin in patients with rheumatoid arthritis)

Craddock, C. G., Longmire, R., AND McMillan, R. (1971) New Engl. J. Med., 285, 324 (Lymphocytes and the immune response)

HANNESTAD, K. (1967) Clin. exp. Immunol., 2, 511 (Presence of aggregated $\gamma$-G globulin in certain rheumatoid synovial effusions)

Hollander, J. L., McCarty, D. J., Astorga, G., And Castro-Murillo, C. (1965) Ann. intern. Med., 62, 271 (Studies on the pathogenesis of rheumatoid joint inflammation. I. The 'RA cell' and a working hypothesis)

Kinsella, T. D. (1973) Clin. exp. Immunol., 14, 187 (Induction of autologous lymphocyte transformation by synovial fluid from patients with rheumatoid arthritis)

- (1974) J. clin. Invest., 53, 1108 (Enhancement of human lymphocyte transformation in vitro by human aggregated gamma globulin)

—, BAUM, J., AND ZIFF, M. (1969) Clin. exp. Immunol., 4, 266 (Immunofluorescent demonstration of an IgG- $\beta$ lc complex in synovial lining cells of rheumatoid synovial membrane)

OuChTERLONY, D. (1962) Progr. Allergy, 6, 30 (Diffusion-in-gel methods for immunological analysis)

ReYNolds, M. D., AND ABDou, N. I. (1973) J. clin. Invest., 52, 1627 (Comparative study of the in vitro proliferative responses of blood and synovial fluid leukocytes of rheumatoid arthritis patients)

Ruddy, S., Gigli, I , AND Austen, K. F. (1972) New Engl. J. Med., 287, 489 (The complement system of man)

SLIWINSKI, A. J., AND ZVAIFLER, N. J. (1970) J. Lab. clin. Med., 76, 304 (In vitro synthesis of IgG by rheumatoid synovium)

SmILEY, J. D., SACHS, C., AND ZIFF, M. (1968) J. clin. Invest., 47, 624 (In vitro synthesis of immunoglobulin by rheumatoid synovial membrane)

Stanfield, A. B., Cubberley, J. B., Parsons, J. L., And Stephens, C. A. L. (1967) Ann. Allergy, 25, 359 (In vitro study of reacted human lymphocytes in perfusion chambers)

Stastny, P., Rosenthal, M., ANDreis, M., AND ZifF, M. (1973) (Abst.) Arthr. and Rheum. 16, 572 (Lymphokines in the rheumatoid joint)

ZIFF, M. (1973) Fed. Proc., 32, 131 (Pathophysiology of rheumatoid arthritis) 